\title{
Exploring the key genes and pathways of osteosarcoma with pulmonary metastasis using a gene expression microarray
}

\author{
ZHONGJU SHI $^{1 *}$, HENGXING ZHOU $^{1 *}$, BIN PAN $^{2 *}$, LU LU $^{1}$, ZHIJIAN WEI $^{1}$, \\ LINLIN SHI $^{1}$, XUE YAO ${ }^{1}$, YI KANG ${ }^{1}$ and SHIQING FENG ${ }^{1}$ \\ ${ }^{1}$ Department of Orthopedics, Tianjin Medical University General Hospital, Tianjin 300052; ${ }^{2}$ Department of Orthopaedics, \\ The Affiliated Hospital of Xuzhou Medical University, Xuzhou, Jiangsu 221006, P.R. China
}

Received November 14, 2016; Accepted June 26, 2017

DOI: $10.3892 / \mathrm{mmr} .2017 .7577$

\begin{abstract}
Osteosarcoma is a common and highly malignant tumour in children and teenagers that is characterized by drug resistance and high metastatic potential. Patients often develop pulmonary metastasis and have a low survival rate. However, the mechanistic basis for pulmonary metastasis remains unclear. To identify key gene and pathways associated with pulmonary metastasis of osteosarcoma, the authors downloaded the gene expression dataset GSE85537 and obtained the differentially expressed genes (DEGs) by analyzing high-throughput gene expression in primary tumours and lung metastases. Subsequently, the authors performed gene ontology (GO) and Kyoto Encyclopedia of Genes and Genomes pathway (KEGG) enrichment analyses and a protein-protein interaction (PPI) network was constructed and analyzed by Cytoscape software. In total, 2,493 genes were identified as DEGs. Of these, 485 genes $(19.45 \%)$ were upregulated, and the remaining 2,008 genes $(80.55 \%)$ were downregulated. The authors identified the predominant GO categories and KEGG pathways that were significantly over-represented in the metastatic OS samples compared with the non-metastatic OS samples. A PPI network was constructed, and the results indicated that ALB, EGFR, INS, IL6, CDH1, FYN, ERBB2, IL8, CXCL12 and RAC2 were the top 10 core genes. The enrichment analyses of the genes involved in the top three significant modules demonstrated that the DEGs were principally related to neuroactive ligand-receptor interaction, the Rap1 signaling pathway, and protein digestion and absorption. Together, these data elucidated the molecular mechanisms of OS patients with pulmonary metastasis and provide potential
\end{abstract}

Correspondence to: Professor Shiqing Feng, Department of Orthopedics, Tianjin Medical University General Hospital, 154 Anshan Road, Heping, Tianjin 300052, P.R. China

E-mail: sqfeng@tmu.edu.cn

*Contributed equally

Key words: osteosarcoma, pulmonary metastasis, microarray, differentially expressed genes, pathways therapeutic targets. However, further experimental studies are needed to confirm these results.

\section{Introduction}

Osteosarcoma (OS) is the most common non-haematological primary bone tumour that affects children and young adolescents, and pulmonary metastases are the major cause of mortality (1-3). In total, $20 \%$ of patients present with pulmonary metastasis at initial diagnosis, and in $\sim 40 \%$ of patients, metastases occur in the advanced stage (4). Furthermore, OS has a low survival rate in patients due to chemoresistance and the high rates of pulmonary metastasis (5). For patients with non-metastatic OS, 5-year OS has increased to 60-70\%, but this rate is reduced to $20 \%$ when metastases occur (6). Therefore, strategies to prevent tumour metastasis are urgently needed. However, the molecular mechanisms of pulmonary metastasis in patients with OS remain poorly understood. Thus, identifying key molecules associated with pulmonary metastasis of OS is important for the development of more effective metastasis-suppressive therapies.

During the last decade, gene expression profiling has identified critical genes and cellular signaling pathways related to the occurrence, development and change of human OS (7). Microarray techniques combined with bioinformatics analysis can determine the differential expression levels of genes accurately and provide an effective tool for large-scale gene expression studies (8). In the patients with OS, the lung is the most common metastatic site, and metastases can lead to a high rate of mortality (9). Therefore, examining differentially expressed genes (DEGs) between non-metastatic and metastatic OS samples is helpful for identifying the key genes and pathways leading to pulmonary metastasis.

In the current study, by comparing the gene expression of non-metastatic and metastatic OS samples in the GEO database, the authors identified DEGs that may be related to pulmonary metastasis. Subsequently, Gene Ontology (GO) and Kyoto Encyclopedia of Genes and Genomes (KEGG) enrichment pathway analyses were performed. In combination with protein-protein interaction (PPI) information, not only did the authors identify relevant genes and pathways, but also revealed existing molecular mechanisms. In conclusion, the authors' finding can improve the understanding of OS and identify 
the genes associated with pulmonary metastasis, thereby providing potential therapeutic targets for further studies.

\section{Materials and methods}

Gene expression microarray data. In the present study, the gene expression profiles of GSE85537 were downloaded from Gene Expression Omnibus (GEO, http://www.ncbi.nlm.nih.gov/geo/). GSE85537 was based on the Affymetrix GPL570 platform (Affymetrix Human Genome U133 Plus 2.0 Array; Affymetrix; Thermo Fisher Scientific, Inc., Waltham, MA, USA). The GSE85537 dataset contained six samples, including three non-metastatic OS samples and three metastatic OS samples.

Identification of DEGs. The raw data files used for the analysis included TXT files. The analysis was carried out using GEO2R (https://www.ncbi.nlm.nih.gov/geo/geo2r/), which can perform comparisons on original submitter-supplied processed data tables using the GEOquery and limma $\mathrm{R}$ packages from the Bioconductor project. The DEGs between the non-metastatic and metastatic OS samples were selected $(\mathrm{P}<0.05)$, and overlapped genes were identified.

GO enrichment and KEGG pathway analysis of the DEGs. After obtaining the DEGs, the authors submitted the DEG list to the online software Database for Annotation, Visualization and Integrated Discovery (DAVID, https://david.ncifcrf. gov/) to identify overrepresented GO categories and pathway categories. GO analysis can determine the biological meaning in a large list of genes and categorize gene product functions, including biological process (BP), molecular function (MF) and cellular component (CC) $(10,11)$. KEGG (http://www.genome. $\mathrm{jp} /$ ) is a knowledge base for systematic analysis of gene functions, linking genomic information with higher-level systemic functions $(12,13)$. Finally, the enriched functions of DEGs were selected via $\mathrm{GO}$ and KEGG pathway analysis, and $\mathrm{P}<0.05$ was considered to indicate a statistically significant difference.

Construction of the PPI Network of DEGs. To further investigate the molecular mechanism of pulmonary metastasis in patients with OS, the Search Tool for the Retrieval of Interacting Genes (STRING) database (http://www.string-db. $\mathrm{org} /$ ) was used to evaluate the interactive relationships among DEGs. Initially, the DEG list was first submitted to STRING, and then, the experimentally validated interactions were selected with a combined score $>0.4$. Subsequently, the PPI networks were analyzed using Cytoscape software (version 3.5.1; www.cytoscape.org). Then, the plug-in Molecular Complex Detection (MCODE) was used to screen the modules of the PPI network in Cytoscape. Furthermore, the enrichment analyses were performed for DEGs in the corresponding modules. $\mathrm{P}<0.05$ was considered to indicate a statistically significant difference.

\section{Results}

Identification of DEGs. The gene expression profile GSE85537 was downloaded from the GEO database, and the GEO2R method was used to identify DEGs in metastatic OS samples compared with non-metastatic OS samples. $\mathrm{P}<0.05$,

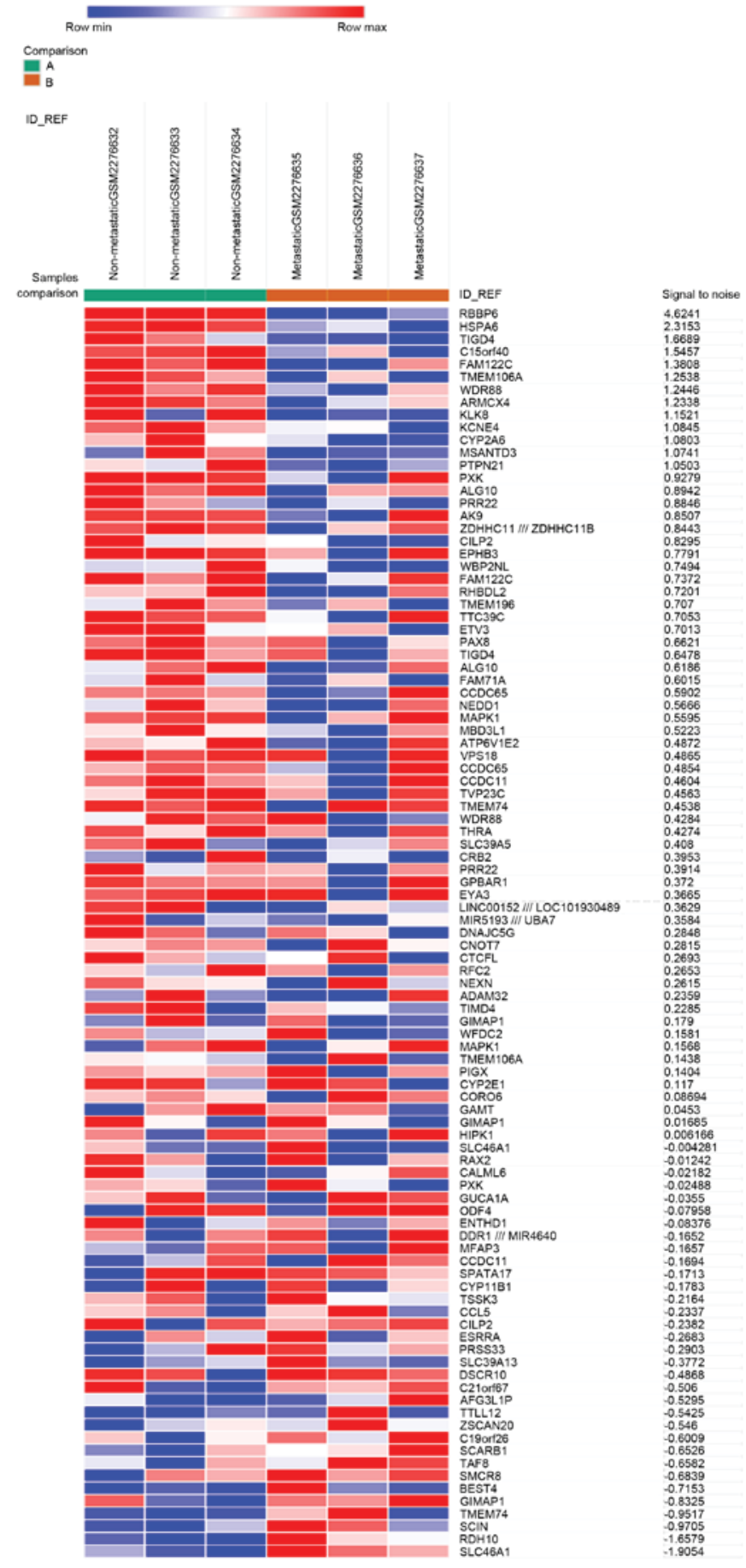

Figure 1 . The heat map of the differentially expressed genes (top 50 upregulated and downregulated genes). Red, upregulation; blue, downregulation.

$\log \mathrm{FC}$ (fold control) $>2.0$ or $\log \mathrm{FC}<-2.0$ was used as the criteria, and 2,493 genes were identified as DEGs. Among these, 485 genes $(19.45 \%)$ were upregulated, and the remaining 2,008 genes $(80.55 \%)$ were downregulated. Subsequently, the top 50 upregulated and downregulated DEGs were selected to generate the heatmap (Fig. 1).

GO term enrichment analysis. To functionally categorize these 2,493 significant genes, the DEGs were analyzed using the online software DAVID. GO analysis revealed that the upregulated DEGs were significantly enriched in BP, including 


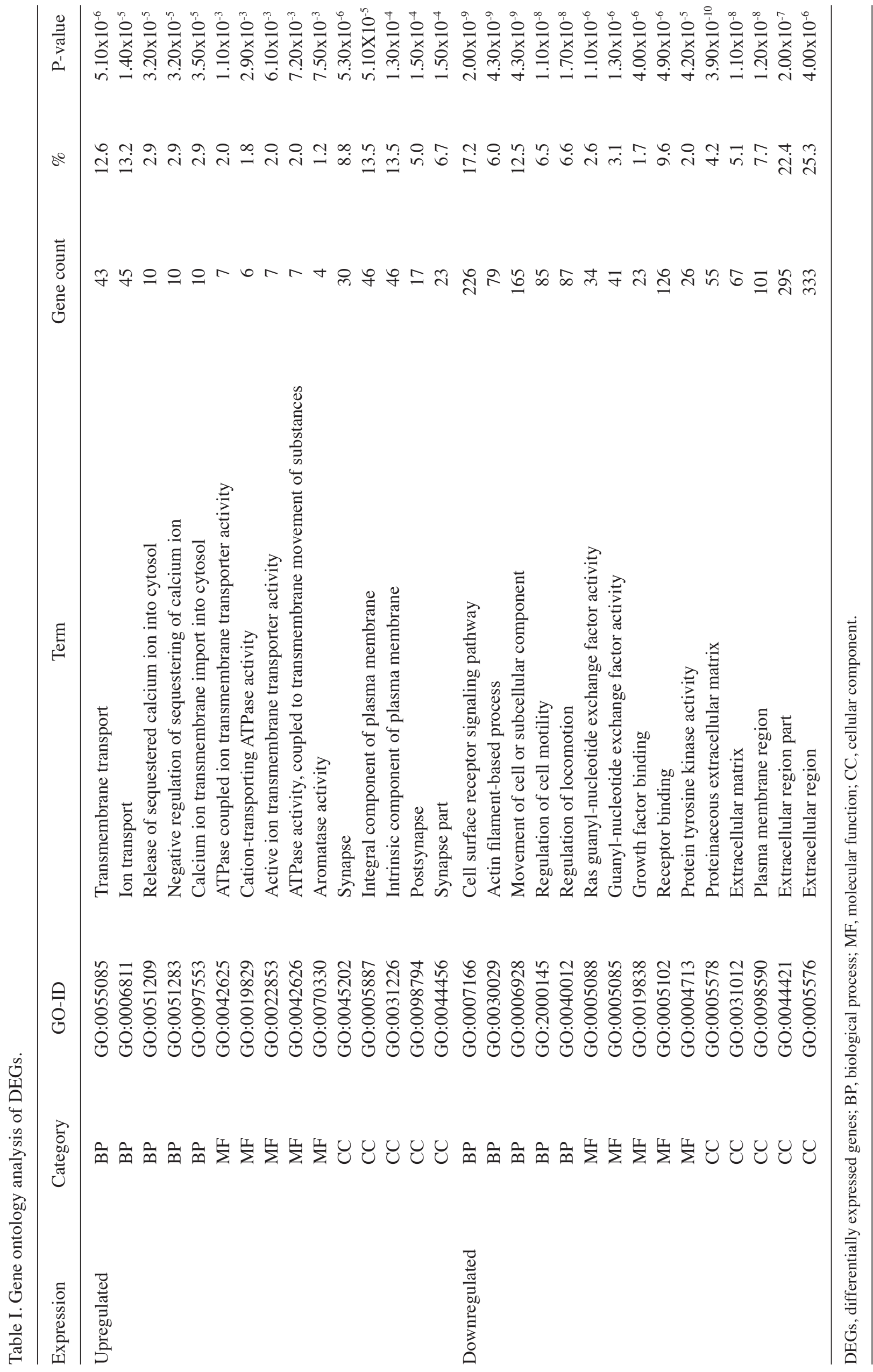




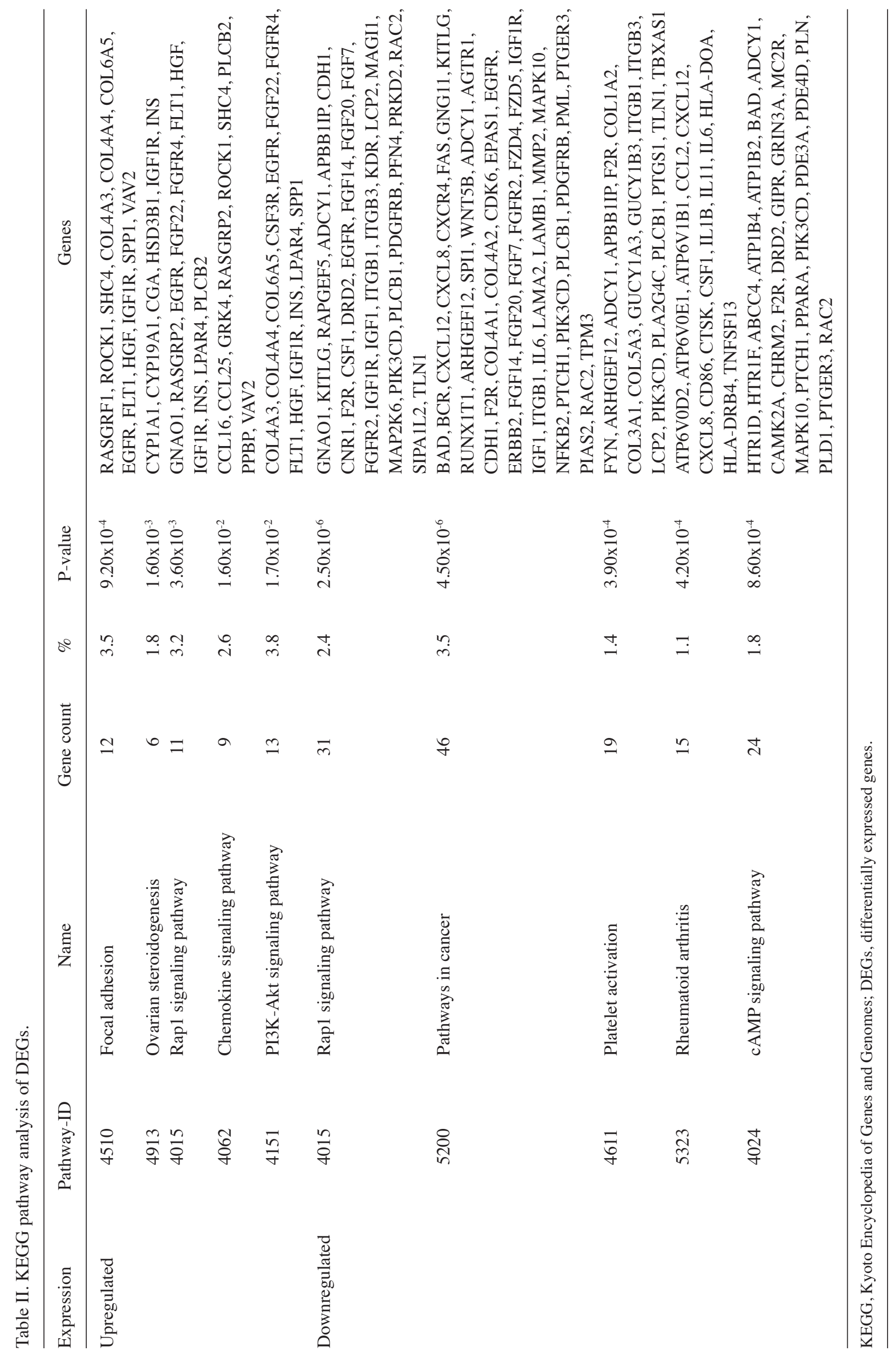


Table III. The core genes and their corresponding degree.

\begin{tabular}{lc}
\hline Gene & Degree \\
\hline ALB & 132 \\
EGFR & 113 \\
INS & 107 \\
IL6 & 77 \\
CDH1 & 76 \\
FYN & 72 \\
ERBB2 & 71 \\
IL8 & 67 \\
CXCL12 & 62 \\
RAC2 & 57 \\
GNAO1 & 55 \\
MMP2 & 54 \\
TOP2A & 54 \\
EDN1 & 53 \\
MYH14 & 52 \\
ITGB1 & 58 \\
ACTA2 & 50 \\
KDR & 50 \\
IGF1 & 59 \\
PPARA & 48 \\
\hline
\end{tabular}

transmembrane transport, ion transport, and release of sequestered calcium ion into cytosol (Table I); the downregulated DEGs were significantly enriched in BP, including cell surface receptor signaling pathway, actin filament-based process and movement of cell or subcellular component (Table I). For MF, the upregulated DEGs were enriched in ATPase-coupled ion transmembrane transporter activity and cation-transporting ATPase activity, and the downregulated DEGs were enriched in Ras guanyl-nucleotide exchange factor activity, guanyl-nucleotide exchange factor activity, and growth factor binding (Table I). In addition, GO CC analysis also indicated that the upregulated DEGs were significantly enriched in the synapse, integral component of plasma membrane and intrinsic component of plasma membrane, and downregulated DEGs were enriched in the proteinaceous extracellular matrix, extracellular matrix and plasma membrane region (Table I).

KEGG pathway analysis. KEGG analysis revealed that the upregulated DEGs were enriched in focal adhesion, ovarian steroidogenesis, Rap1 signaling pathway, chemokine signaling pathway and PI3K-Akt signaling pathway, while the downregulated DEGs were enriched in the Rapl signaling pathway, pathways in cancer, platelet activation, rheumatoid arthritis and cAMP signaling pathway (Table II).

PPI network of the DEGs and core genes in the PPI network. Based on the information in the STRING database, the PPI network contained 1,123 nodes and 4,986 edges. The nodes indicate the DEGs, and the edges indicate the interactions between the DEGs. The top 10 high-degree hub nodes included albumin (ALB), epidermal growth factor receptor (EGFR), insulin (INS), interleukin 6 (IL-6), cadherin 1
(CDH1), FYN proto-oncogene (FYN), erb-b2 receptor tyrosine kinase 2 (ERBB2), interleukin 8 (IL8), C-X-C motif chemokine ligand 12 (CXCL12) and Ras-related C3 botulinum toxin substrate 2 (RAC2). Among these genes, ALB presented the highest node degree, which was 132 . The core genes and their corresponding degree are shown in Table III. Then, the authors used MCODE to screen the modules of the PPI network (Fig. 2), and performed an enrichment analysis of the genes involved in the top three significant modules. The results demonstrated that the DEGs in modules 1-3 were principally related to neuroactive ligand-receptor interaction, the Rap1 signaling pathway, and protein digestion and absorption (Table IV).

\section{Discussion}

Osteosarcoma is the most common primary malignant bone tumour, is commonly observed in children and adolescents and shows a strong tendency for pulmonary metastasis (14). Pulmonary metastasis can cause medical therapy failure and high mortality rate in osteosarcoma patients $(15,16)$. However, the present knowledge of the molecular mechanism of pulmonary metastasis in patients with OS remains insufficient. Therefore, the elucidation of molecular mechanisms to inhibit the metastasis of OS is imperative. High-throughput profiling technologies, such as microarrays, have been widely used and regarded as invaluable tools to identify potential therapeutic targets $(17,18)$. The present study performed a comprehensive analysis and built a gene interaction network based on the gene expression profiles (GSE85537), comprising three non-metastatic OS samples and three metastatic OS samples. The results of the analysis demonstrated that there were 2,493 DEGs in the metastatic OS tumour samples compared with non-metastatic OS tumour samples, and among these DEGs, 485 were upregulated and 2,008 were downregulated. Moreover, GO and KEGG pathway analyses were performed to obtain a better understanding of the interactions of DEGs. Combining with the PPI network, key potential genes and pathways that may be associated with pulmonary metastasis of OS were identified.

The results of GO analyses indicated that the significant ontology categories included transmembrane transport, ion transport and aromatase activity. The system of transport is essential to every living cell and it had multiple functions, including allowing the entry to all essential molecules, exporting macromolecules such as proteins and DNA, providing cellular concentrations of ions $(19,20)$. In a recent study on papillary thyroid carcinoma progression, downregulated DEGs were also predominantly enriched in transmembrane transport process (21). Additionally, ion transport system also stimulates the progression of pithelial-mesenchymal transition (EMT) in cervical carcinoma cells (22). Therefore, transmembrane transport and ion transport are fundamental processes and may serve roles in pulmonary metastasis of OS. Moreover, aromatase is an enzyme that converts testosterone into estradiol, and the control of aromatase activity mainly depends on $\mathrm{Ca}^{2+}$ transients (23). It has been reported that aromatase activity is associated with tumour-node-metastasis staging in human breast cancer (24). Cell motility is essential in the regulation of cancer progression, and aberrant cell motility occurs in 
Table IV. The enriched pathways of modules.

\begin{tabular}{|c|c|c|c|c|}
\hline Modules & Enriched pathways & P-value & $\begin{array}{l}\text { False } \\
\text { discovery rate }\end{array}$ & Nodes \\
\hline \multirow[t]{3}{*}{1} & $\begin{array}{l}\text { Neuroactive ligand- } \\
\text { receptor interaction }\end{array}$ & $9.60 \times 10^{-10}$ & $2.68 \times 10^{-14}$ & $\begin{array}{l}\text { ADORA3, CHRM2, CHRM4, CNR1, DRD2, GRM3, } \\
\text { GRM6, HTR1D, HTR1F, PTGER3, S1PR1, S1PR5 }\end{array}$ \\
\hline & Chemokine signaling & $6.10 \times 10^{-6}$ & $2.03 \times 10^{-7}$ & CCL16, CCL25, CXCL12, CXCL2, CXCR4, IL8, PPBP \\
\hline & Cytokine-cytokine & $5.00 \times 10^{-4}$ & $1.87 \times 10^{-6}$ & CCL16, CCL25, CXCL12, CXCL2, CXCR4, IL8, PPBP \\
\hline \multirow[t]{3}{*}{2} & $\begin{array}{l}\text { Rap1 signaling } \\
\text { pathway }\end{array}$ & $2.40 \times 10-{ }^{16}$ & $5.46 \times 10^{-21}$ & $\begin{array}{l}\text { CDH1, EGFR, F2R, FGF7, FLT1, GNAO1, HGF, IGF1, } \\
\text { IGF1R, INS, KDR, LPAR4, PDGFRB, PLCB1, PLCB2 }\end{array}$ \\
\hline & $\begin{array}{l}\text { PI3K-Akt signaling } \\
\text { pathway }\end{array}$ & $3.20 \times 10^{-9}$ & $1.09 \times 10^{-12}$ & $\begin{array}{l}\text { EGFR, F2R, FGF7, FLT1, HGF, IGF1, IGF1R, IL6, INS, } \\
\text { KDR, LPAR4, PDGFRB }\end{array}$ \\
\hline & $\begin{array}{l}\text { Calcium signaling } \\
\text { pathway }\end{array}$ & $6.10 \times 10^{-8}$ & $1.09 \times 10^{-10}$ & $\begin{array}{l}\text { AGTR1, EGFR, ERBB2, F2R, GRPR, PDGFRB, } \\
\text { PLCB1, PLCB2, PTGFR }\end{array}$ \\
\hline \multirow[t]{3}{*}{3} & $\begin{array}{l}\text { Protein digestion } \\
\text { and absorption }\end{array}$ & $4.30 \times 10^{-14}$ & $1.48 \times 10^{-16}$ & $\begin{array}{l}\text { COL1A2, COL21A1, COL3A1, COL4A1, COL4A2, } \\
\text { COL4A3, COL4A4, COL5A3 }\end{array}$ \\
\hline & $\begin{array}{l}\text { ECM-receptor } \\
\text { interaction }\end{array}$ & $2.30 \times 10^{-11}$ & $8.52 \times 10^{-14}$ & $\begin{array}{l}\text { COL1A2, COL3A1, COL4A1, COL4A2, COL4A } 3 \text {, } \\
\text { COL4A4, COL5A3 }\end{array}$ \\
\hline & Amoebiasis & $7.80 \times 10^{-11}$ & $2.44 \times 10^{-13}$ & $\begin{array}{l}\text { COL1A2, COL3A1, COL4A1, COL4A2, COL4A3, } \\
\text { COL4A4, COL5A3 }\end{array}$ \\
\hline
\end{tabular}
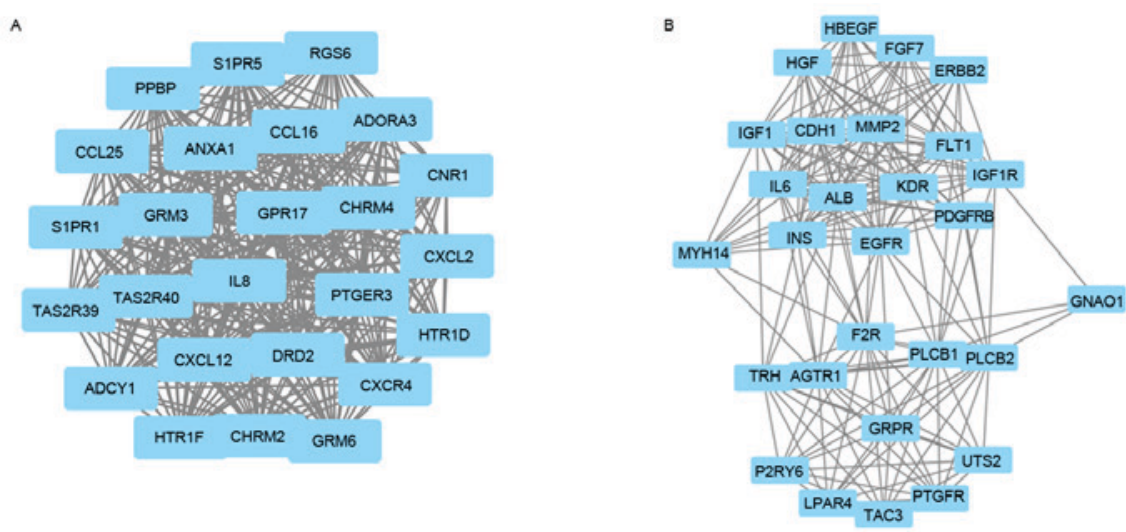

$c$

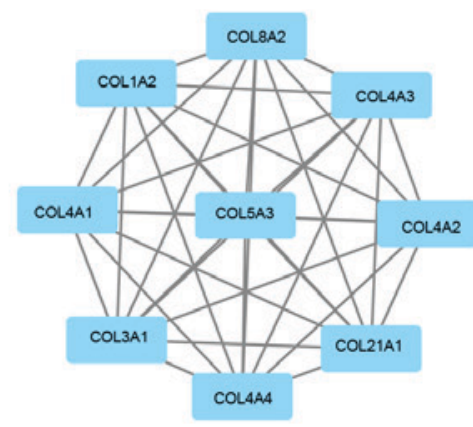

Figure 2. Top three modules from the protein-protein interaction network. (A) Module 1, (B) module 2, (C) module 3.

malignant cancers and results in tumour metastasis (25). The activation of ERBB3-dependent signaling serves a key role in the regulation of cell motility, and regulation of cell motility contributes to intrahepatic metastasis and early recurrence of hepatocellular carcinoma (HCC) (26). Cell motility is also considered as an important process in the regulation of metastasis of oral cancer (27). Cell motility may therefore be important to pulmonary metastasis of OS. In addition, many studies have demonstrated that receptor binding is closely correlated with tumour metastasis, and these receptor bindings include fibroblast growth factor and vascular endothelial growth factor receptor binding, duffy antigen receptor for chemokines and growth factor receptor bound protein $2 \mathrm{Src}$ homology 2 domain binding (28-30). Therefore, GO analyses can help identify the possible biological processes, molecular functions and cellular components involved in tumour metastasis.

Moreover, the KEGG pathways revealed that DEGs were enriched in focal adhesion, platelet activation, the Rap1 signaling pathway and the PI3K-Akt signaling pathway. Focal adhesion is a dynamic multi-protein complex that connects intracellular actin fibres and extracellular substrates $(31,32)$. Focal adhesion kinase (FAK) is an effective therapeutic target of cell migration in various tumour cells (33-35). Furthermore, Rap1 is considered as a protein that can revert Ras transformation, and it is a key mediator in the control of integrin activation (36). A recent study revealed that the cAMP/Epac/Rap1 signaling cascade had a crucial role in blood-tumour barrier hyperpermeability (37). Cancer cell-induced platelet activation is identified as a critical event responsible for prometastatic activity of platelets, and blocking platelet aggregation can inhibit the progression of skeletal metastases, yet the mechanism underlying this effect is still unknown (38). Moreover, the PI3 K/Akt signaling pathway is involved in various cellular processes, such as inflammation, autophagy and cancer progression (39), and many studies have demonstrated that activation of the PI3K-Akt signaling pathway can increase the metastatic potential (40-42). 
Therefore, further understanding of these signaling pathways can help us to elucidate the crucial mechanism of tumour metastasis.

Furthermore, the authors analyzed the PPI network and found that ALB, EGFR, INS, IL6, CDH1, FYN, ERBB2, IL8, CXCL12 and RAC2 were the top 10 core genes, which may be potential therapeutic targets for pulmonary metastasis of OS. Node degree refers to the number of interacting partners per protein. Among these genes, ALB showed the highest node degree. Albumin is one of the major plasma proteins and is synthesized and secreted primarily by hepatocytes, and it is often used for diagnosis of HCC (43). Albumin is a major indicator of a favourable outcome of HCC, and it can suppress cell proliferation via reduction of phosphorylation of $\mathrm{Rb}$ (retinoblastoma) proteins and increase of p21 and p57 (44). A recent study indicated that the expression of albumin increased in human hepatoma BEL-7402 cells after bufalin was used, and ALB may be associated with bufalin-mediated inhibition of the invasion and metastasis of HCC cells (45). Albumin mRNA was also found in lung by means of reverse-transcription polymerase chain reaction, so albumin may serve a role in pulmonary metastasis (46). The present study indicated that ALB was a key gene with the highest node degree in OS, therefore ALB may be a potential target for inhibiting OS tumour metastasis, but further experimental verification is necessary.

The EGFR is a key component in the mitogen-activated protein kinase (MAPK) pathway, and anti-EGFR therapy can lead to inhibition of tumour growth, invasion and metastasis via inhibition of the MAPK pathway (47). Moreover, nasopharyngeal carcinoma (NPC), which has the highest rate of metastasis, has been extensively studied, and inhibition of EGFR can inhibit NPC cell migration and invasion (48). Additionally, EGFR has been shown to promote survival of prostate tumour-initiating cells and circulating tumour cells that metastasize to bone (49). These results corresponded with the function analysis in the present study, and EGFR is likely to be an important regulated target in pulmonary metastasis of OS. Therefore, EGFR may be a potential therapeutic target and prognostic indicator in OS patients, and a thorough study of EGFR in OS is needed. Furthermore, a recent study demonstrated that inflammatory cytokines, such as IL-6, promoted proliferation, migration, invasion and EMT of gallbladder cancer both in vitro and in vivo (50). Overexpression of human epidermal growth factor receptor 2 (ErbB2 or HER2) occurs in $\sim 30 \%$ of breast cancer patients, and overexpression of activated ErbB2 in the mammary epithelium can lead to the rapid induction of metastatic multifocal mammary tumours $(51,52)$. Therefore, these core genes in the current study have been suggested to be associated with tumour metastasis in the previous studies, so the analysis of these core genes is useful for understanding the molecular mechanisms and identifying therapeutic targets of OS with pulmonary metastasis. However, subsequent prospective studies will be required to further confirm the function of these core genes in pulmonary metastasis of OS.

Module analysis of the PPI network demonstrated that the pulmonary metastasis of OS was associated with neuroactive ligand-receptor interaction, the Rap1 signaling pathway, protein digestion and absorption and other processes. It has been demonstrated that neuroactive ligand-receptor interaction is important in HCC progression and it is present in the early-, middle- and late-stages of $\operatorname{HCC}(53,54)$. Moreover, the neuroactive ligand-receptor interaction pathway was enriched in prostate tumours from African-American patients (55). Therefore, neuroactive ligand-receptor interaction appears to be important in pulmonary metastasis of OS. Moreover, the protein digestion and absorption pathway has been reported to be associated with pancreatic neuroendocrine tumours and breast cancer $(56,57)$. Thus, the roles of these pathways in pulmonary metastasis of OS need to be confirmed by the future studies, and these results may lead to additional therapeutic alternatives in the patients with OS.

In conclusion, the present study identified 2,493 DEGs, which may be involved in the progress of pulmonary metastasis in OS patients, via a comprehensive bioinformatics analysis. GO term, KEGG pathway and PPI network analyses provided a set of related genes and pathways to help elucidate the molecular mechanisms of pulmonary metastasis. Further experimental studies are needed to confirm these results and should help determine potential targets for inhibiting pulmonary metastasis in OS patients.

\section{Acknowledgements}

The present study was supported by the State Key Program of National Natural Science Foundation of China (grant no. 81330042), International Cooperation Program of National Natural Science Foundation of China (grant no. 81620108018), Special Program for Sino-Russian Joint Research Sponsored by the Ministry of Science and Technology, China (grant no. 2014DFR31210) and Key Program Sponsored by the Tianjin Science and Technology Committee, China (grant nos. 13RCGFSY19000 and 14ZCZDSY00044).

\section{References}

1. Klein MJ and Siegal GP: Osteosarcoma: Anatomic and histologic variants. Am J Clin Pathol 125: 555-581, 2006.

2. Neklyudova O, Arlt MJ, Brennecke P, Thelen M, Gvozdenovic A, Kuzmanov A, Robl B, Botter SM, Born W and Fuchs B: Altered CXCL12 expression reveals a dual role of CXCR4 in osteosarcoma primary tumor growth and metastasis. J Cancer Res Clin Oncol 142: 1739-1750, 2016.

3. Zandueta C, Ormazábal C, Perurena N, Martínez-Canarias S, Zalacaín M, Julián MS, Grigoriadis AE, Valencia K, Campos-Laborie FJ, Rivas Jde L, et al: Matrix-Gla protein promotes osteosarcoma lung metastasis and associates with poor prognosis. J Pathol 239: 438-449, 2016.

4. Qu L and Liu B: Cyclooxygeanse-2 promotes metastasis in osteosarcoma. Cancer Cell Int 15: 69, 2015.

5. Weekes D, Kashima TG, Zandueta C, Perurena N, Thomas DP, Sunters A, Vuillier C, Bozec A, El-Emir E, Miletich I, et al: Regulation of osteosarcoma cell lung metastasis by the c-Fos/AP-1 target FGFR1. Oncogene 35: 2852-2861, 2016.

6. Gorlick R, Anderson P, Andrulis I, Arndt C, Beardsley GP, Bernstein M, Bridge J, Cheung NK, Dome JS, Ebb D et al: Biology of childhood osteogenic sarcoma and potential targets for therapeutic development: Meeting summary. Clin Cancer Res 9: 5442-5453, 2003.

7. Selvarajah GT, Kirpensteijn J, van Wolferen ME, Rao NA, Fieten $\mathrm{H}$ and Mol JA: Gene expression profiling of canine osteosarcoma reveals genes associated with short and long survival times. Mol Cancer 8: 72, 2009.

8. Wolf M, El-Rifai W, Tarkkanen M, Kononen J, Serra M, Eriksen EF, Elomaa I, Kallioniemi A, Kallioniemi OP and Knuutila S: Novel findings in gene expression detected in human osteosarcoma by cDNA microarray. Cancer Genet Cytogenet 123: 128-132, 2000. 
9. Zhang N, Xie T, Xian M, Wang YJ, Li HY, Ying MD and Ye ZM: SIRT1 promotes metastasis of human osteosarcoma cells. Oncotarget 7: 79654-79669, 2016.

10. Ashburner M, Ball CA, Blake JA, Botstein D, Butler H, Cherry JM, Davis AP, Dolinski K, Dwight SS, Eppig JT, et al: Gene ontology: Tool for the unification of biology. The Gene Ontology Consortium. Nat Genet 25: 25-29, 2000.

11. Lammers G, Gilissen C, Nillesen ST, Uijtdewilligen PJ, Wismans RG, Veltman JA, Daamen WF and van Kuppevelt TH: High density gene expression microarrays and gene ontology analysis for identifying processes in implanted tissue engineering constructs. Biomaterials 31: 8299-8312, 2010.

12. Kanehisa M and Goto S: KEGG: Kyoto encyclopedia of genes and genomes. Nucleic Acids Res 28: 27-30, 2000.

13. Wixon J and Kell D: The Kyoto encyclopedia of genes and genomes-KEGG. Yeast 17: 48-55, 2000.

14. Lu J, Song G, Tang Q, Zou C, Han F, Zhao Z, Yong B, Yin J, $\mathrm{Xu} \mathrm{H}, \mathrm{Xie} \mathrm{X}$, et al: IRX1 hypomethylation promotes osteosarcoma metastasis via induction of CXCL14/NF- $\kappa \mathrm{B}$ signaling. J Clin Invest 125: 1839-1856, 2015.

15. Isakoff MS, Bielack SS, Meltzer P and Gorlick R: Osteosarcoma: Current treatment and a collaborative pathway to success. J Clin Oncol 33: 3029-3035, 2015.

16. Siegel HJ and Pressey JG: Current concepts on the surgical and medical management of osteosarcoma. Expert Rev Anticancer Ther 8: 1257-1269, 2008

17. Liang B, Li C and Zhao J: Identification of key pathways and genes in colorectal cancer using bioinformatics analysis. Med Oncol 33: 111, 2016

18. Yin F, Shu L, Liu X, Li T, Peng T, Nan Y, Li S, Zeng X and Qiu X: Microarray-based identification of genes associated with cancer progression and prognosis in hepatocellular carcinoma. J Exp Clin Cancer Res 35: 127, 2016.

19. Hollenstein K, Dawson RJ and Locher KP: Structure and mechanism of ABC transporter proteins. Curr Opin Struct Biol 17: 412-418, 2007.

20. Yen MR, Choi J and Saier MH Jr: Bioinformatic analyses of transmembrane transport: Novel software for deducing protein phylogeny, topology and evolution. J Mol Microbiol Biotechnol 17: 163-176, 2009.

21. Qiu J, Zhang W, Xia Q, Liu F, Li L, Zhao S, Gao X, Zang C, Ge R and Sun Y: RNA sequencing identifies crucial genes in papillary thyroid carcinoma (PTC) progression. Exp Mol Pathol 100: 151-159, 2016.

22. Lee MY and Shen MR: Epithelial-mesenchymal transition in cervical carcinoma. Am J Transl Res 4: 1-13, 2012.

23. Fester L, Zhou L, Ossig C, Labitzke J, Bläute C, Bader M, Vollmer G, Jarry H and Rune GM: Synaptopodin is regulated by aromatase activity. J Neurochem 140: 126-139, 2017.

24. Bolufer P, Ricart E, Lluch A, Vazquez C, Rodriguez A, Ruiz A, Llopis F, Garcia-Conde J and Romero R: Aromatase activity and estradiol in human breast cancer: Its relationship to estradiol and epidermal growth factor receptors and to tumor-node-metastasis staging. J Clin Oncol 10: 438-446, 1992.

25. Hammer A and Diakonova M: Prolactin-induced PAK1 tyrosyl phosphorylation promotes FAK dephosphorylation, breast cancer cell motility, invasion and metastasis. BMC Cell Biol 17: 31, 2016.

26. Hsieh SY, He JR, Hsu CY, Chen WJ, Bera R, Lin KY, Shih TC, Yu MC, Lin YJ, Chang CJ, et al: Neuregulin/erythroblastic leukemia viral oncogene homolog 3 autocrine loop contributes to invasion and early recurrence of human hepatoma. Hepatology 53: 504-516, 2011.

27. Noguti J, De Moura CF, De Jesus GP, Da Silva VH, Hossaka TA, Oshima CT and Ribeiro DA: Metastasis from oral cancer: An overview. Cancer Genomics Proteomics 9: 329-335, 2012.

28. Aviezer D, Cotton S, David M, Segev A, Khaselev N, Galili N, Gross Z and Yayon A: Porphyrin analogues as novel antagonists of fibroblast growth factor and vascular endothelial growth factor receptor binding that inhibit endothelial cell proliferation, tumor progression, and metastasis. Cancer Res 60: 2973-2980, 2000.

29. Giubellino A, Gao Y, Lee S, Lee MJ, Vasselli JR, Medepalli S, Trepel JB, Burke TR Jr and Bottaro DP: Inhibition of tumor metastasis by a growth factor receptor bound protein $2 \mathrm{Src}$ homology 2 domain-binding antagonist. Cancer Res 67 : 6012-6016, 2007.

30. Wang J, Ou ZL, Hou YF, Luo JM, Shen ZZ, Ding J and Shao ZM: Enhanced expression of Duffy antigen receptor for chemokines by breast cancer cells attenuates growth and metastasis potential. Oncogene 25: 7201-7211, 2006.
31. Case LB and Waterman CM: Integration of actin dynamics and cell adhesion by a three-dimensional, mechanosensitive molecular clutch. Nat Cell Biol 17: 955-963, 2015.

32. Sulzmaier FJ, Jean C and Schlaepfer DD: FAK in cancer: Mechanistic findings and clinical applications. Nat Rev Cancer 14: 598-610, 2014.

33. Kishi T, Mayanagi T, Iwabuchi S, Akasaka T and Sobue K Myocardin-related transcription factor A (MRTF-A) activity-dependent cell adhesion is correlated to focal adhesion kinase (FAK) activity. Oncotarget 7: 72113-72130, 2016.

34. Mendoza P, Ortiz R, Díaz J, Quest AF, Leyton L, Stupack D and Torres VA: Rab5 activation promotes focal adhesion disassembly, migration and invasiveness in tumor cells. J Cell Sci 126: 3835-3847, 2013

35. Salem I, Alsalahi M, Chervoneva I, Aburto LD, Addya S, Ott GR, Ruggeri BA, Cristofanilli M and Fernandez SV: The effects of CEP-37440, an inhibitor of focal adhesion kinase, in vitro and in vivo on inflammatory breast cancer cells. Breast Cancer Res 18: 37, 2016

36. Riedl JA, Brandt DT, Batlle E, Price LS, Clevers H and Bos JL: Down-regulation of Rap1 activity is involved in ephrinB1-induced cell contraction. Biochem J 389: 465-469, 2005.

37. Li Z, Liu XB, Liu YH, Xue YX, Wang P, Liu LB, Yao YL and Ma J: Functions for the cAMP/Epac/Rap1 signaling pathway in low-dose endothelial monocyte-activating polypeptide-II-induced opening of blood-tumor barrier. J Mol Neurosci 57: 1-10, 2015.

38. Leblanc R and Peyruchaud O: The role of platelets and megakaryocytes in bone metastasis. J Bone Oncol 5: 109-111, 2016.

39. Chen XS, Li LY, Guan YD, Yang JM and Cheng Y: Anticancer strategies based on the metabolic profile of tumor cells: Therapeutic targeting of the Warburg effect. Acta Pharmacol Sin 37: 1013-1019, 2016.

40. Cohen-Solal KA, Boregowda RK and Lasfar A: RUNX2 and the $\mathrm{PI} 3 \mathrm{~K} / \mathrm{AKT}$ axis reciprocal activation as a driving force for tumor progression. Mol Cancer 14: 137, 2015.

41. Liu JF, Tsao YT and Hou CH: Amphiregulin enhances intercellular adhesion molecule-1 expression and promotes tumor metastasis in human osteosarcoma. Oncotarget 6: 40880-40895, 2015.

42. Yang Y, Gao Z, Ma Y, Teng H, Liu Z, Wei H, Lu Y, Cheng X, Hou L and Zou X: Fucoidan inhibits lymphangiogenesis by downregulating the expression of VEGFR3 and PROX1 in human lymphatic endothelial cells. Oncotarget 7: 38025-38035, 2016.

43. Ohguchi S, Nakatsukasa H, Higashi T, Ashida K, Nouso K, Ishizaki M, Hino N, Kobayashi Y, Uematsu S and Tsuji T: Expression of alpha-fetoprotein and albumin genes in human hepatocellular carcinomas: Limitations in the application of the genes for targeting human hepatocellular carcinoma in gene therapy. Hepatology 27: 599-607, 1998

44. Nojiri S and Joh T: Albumin suppresses human hepatocellular carcinoma proliferation and the cell cycle. Int J Mol Sci 15: 5163-5174, 2014

45. Gai JQ, Sheng X, Qin JM, Sun K, Zhao W and Ni L: The effect and mechanism of bufalin on regulating hepatocellular carcinoma cell invasion and metastasis via Wnt/ $\beta$-catenin signaling pathway. Int J Oncol 48: 338-348, 2016.

46. Chou HC, Sheu JC, Huang GT, Wang JT and Chen DS: Albumin messenger RNA is not specific for circulating hepatoma cells. Gastroenterology 107: 630-631, 1994.

47. Yang J, Li S, Wang B, Wu Y, Chen Z, Lv M, Lin Y and Yang J: Potential biomarkers for anti-EGFR therapy in metastatic colorectal cancer. Tumour Biol 37: 11645-11655, 2016.

48. Zheng LS, Yang JP, Cao Y, Peng LX, Sun R, Xie P, Wang MY, Meng DF, Luo DH, Zou X, et al: SPINK6 promotes metastasis of nasopharyngeal carcinoma via binding and activation of epithelial growth factor receptor. Cancer Res 77: 579-589, 2017.

49. Day KC, Hiles GL, Kozminsky M, Dawsey SJ, Paul A, Broses LJ, Shah R, Kunja LP, Hall C, Palanisamy N, et al: HER2 and EGFR overexpression support metastatic progression of prostate cancer to bone. Cancer Res 77: 74-85, 2017.

50. Zhang M, Gong W, Zuo B, Chu B, Tang Z, Zhang Y, Yang Y, Zhou D, Weng M, Qin Y, et al: The microRNA miR-33a suppresses IL-6-induced tumor progression by binding Twist in gallbladder cancer. Oncotarget 7: 78640-78652, 2016.

51. Bertucci F, Finetti P, Guille A, Adélaïde J, Garnier S, Carbuccia N, Monneur A, Charafe-Jauffret E, Goncalves A, Viens P, et al: Comparative genomic analysis of primary tumors and metastases in breast cancer. Oncotarget 7: 27208-27219, 2016. 
52. Schade B, Lesurf R, Sanguin-Gendreau V, Bui T, Deblois G, O'Toole SA, Millar EK, Zardawi SJ, Lopez-Knowles E, Sutherland RL, et al: $\beta$-Catenin signaling is a critical event in ErbB2-mediated mammary tumor progression. Cancer Res 73: 4474-4487, 2013.

53. Zhang MH, Shen QH, Qin ZM, Wang QL and Chen X: Systematic tracking of disrupted modules identifies significant genes and pathways in hepatocellular carcinoma. Oncol Lett 12: 3285-3295, 2016.

54. Zhao Y, Xue F, Sun J, Guo S, Zhang H, Qiu B, Geng J, Gu J, Zhou X, Wang W, et al: Genome-wide methylation profiling of the different stages of hepatitis B virus-related hepatocellular carcinoma development in plasma cell-free DNA reveals potential biomarkers for early detection and high-risk monitoring of hepatocellular carcinoma. Clin Epigenetics 6: 30, 2014.
55. Myers JS, von Lersner AK and Sang QX: Proteomic upregulation of fatty acid synthase and fatty acid binding protein 5 and identification of cancer- and race-specific pathway associations in human prostate cancer tissues. J Cancer 7: 1452-1464, 2016.

56. Akkiprik M, Peker I, Özmen T, Amuran GG, Güllüoğlu BM, Kaya $\mathrm{H}$ and Özer A: Identification of differentially expressed IGFBP5-related genes in breast cancer tumor tissues using cDNA microarray experiments. Genes (Basel) 6: 1201-1214, 2015.

57. Wang DD, Liu ZW, Han MM, Zhu ZM, Tu YL, Dou CQ, Jin X, Cai SW and Du N: Microarray based analysis of gene expression patterns in pancreatic neuroendocrine tumors. Eur Rev Med Pharmacol Sci 19: 3367-3374, 2015. 